\title{
Matemáticas
}

\section{de alto vuelo}

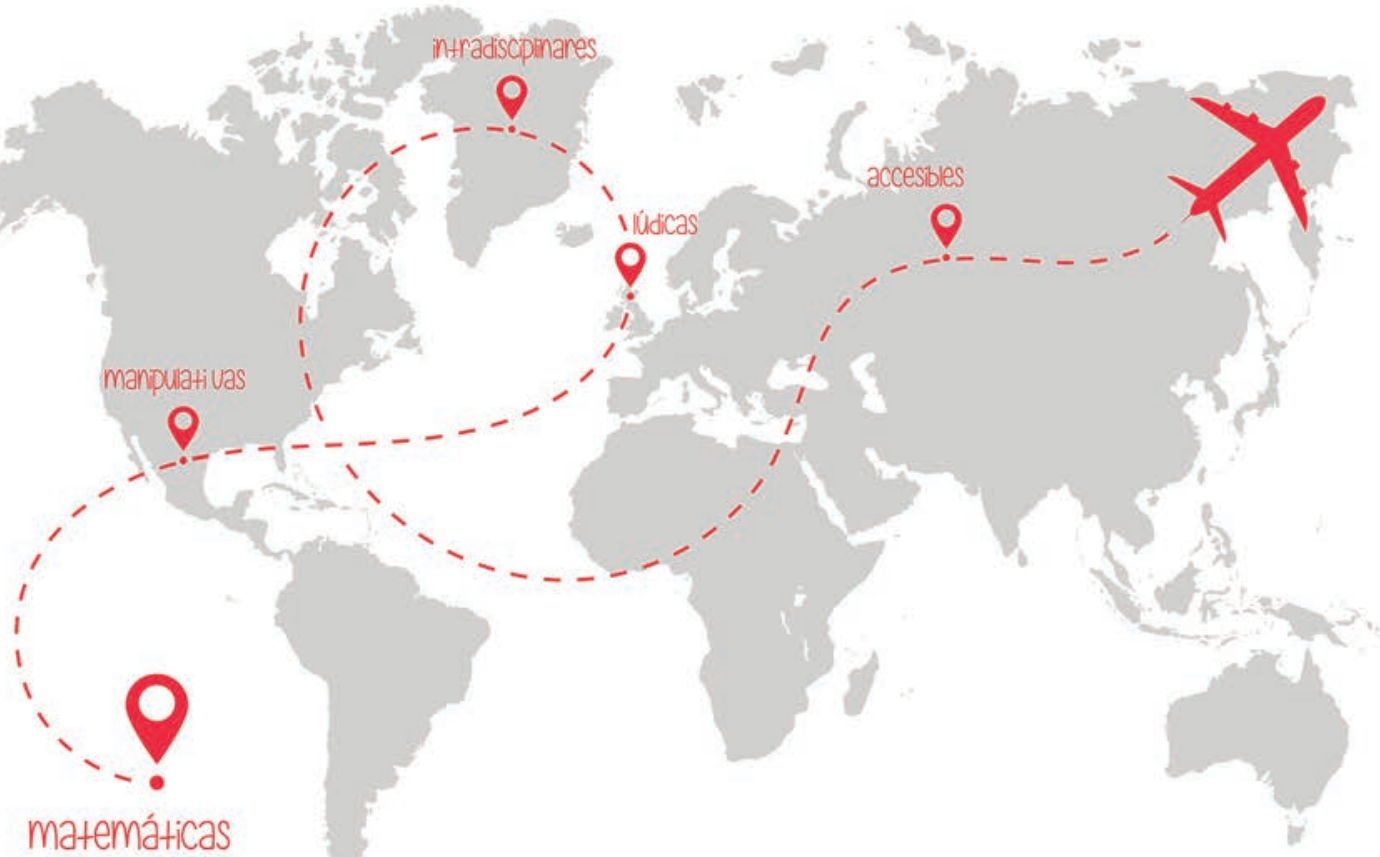

Como si de un viaje en avión se tratara, Pablo y Clara, dos alumnos de Educación Primaria de la Universidad Pontificia Comillas, relatan su proceso de aprendizaje en relación con la didáctica de las

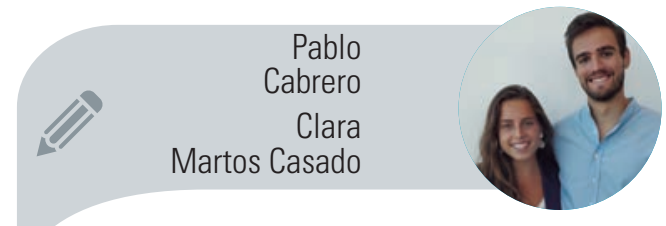

Estudiantes de Doble Grado Educación Primaria + Educación Infantil en Universidad Pontificia Comillas pablo.cabrero.sanchez@gmail.com martos.clara@gmail.com

Matemáticas. Ponen de relieve los aspectos

que consideran fundamentales para una buena enseñanza y su consecuente valioso aprendizaje. Además, comparten con los lectores diferentes experiencias de aprendizaje vividas durante sus años de formación. 


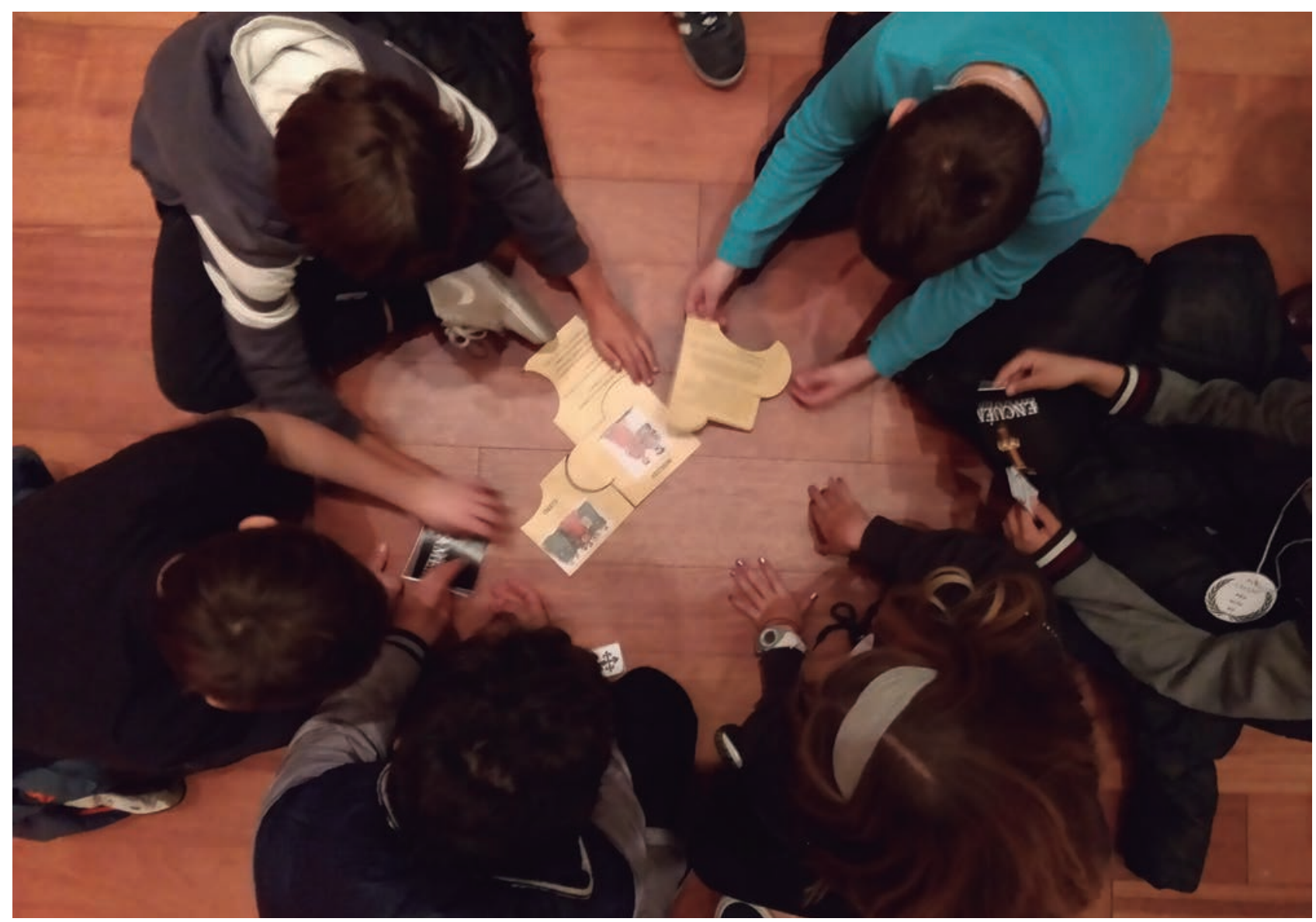

Buenos días señores y señoras pasajeros, la comandante Santaolalla y la tripulación, en nombre de Comillas Airlines, les damos la bienvenida a bordo de este vuelo. Les advertimos de que comienzan un trayecto con un destino aún por decidir. Por favor, hagan uso de los materiales manipulativos, pongan los cartones del Numerator en la posición correcta y utilicen la calculadora siempre que sea necesario. Les recordamos que en todos los vuelos de esta aerolínea están prohibidos los "porque sí" y las matemáticas mecanizadas sin sentido. Prepárense para el despegue.

Antes de despegar nos gustaría presentarnos: somos Pablo y Clara, estudiantes de $3 .^{\circ}$ de Educación Primaria e Infantil de la Universidad Pontificia Comillas. Es para nosotros un reto y, a la vez, un orgullo, participar en este monográfico de la revista en el que colaboran autores de la talla de Ángel Alsina, protagonista de muchos de nuestros aprendizajes a lo largo de este curso.

Para afrontar este reto hemos querido dar rienda suelta a nuestra creatividad a través de una metáfora que comparase nuestro proceso de formación como maestros de matemáticas con el transcurso de un vuelo. Por lo tanto, hablaremos de maletas, álbumes de fotos o escalas en vez de aprendizaje, blogs de clase o proyectos interdisciplinares. Esperamos que os guste.

\section{Nunca una pérdida había supuesto tanta ganancia}

Antes de emprender cualquier viaje hay que hacer una buena maleta. Los más metódicos utilizan una lista de qué llevar para no olvidarse de nada, y hay también quien abre el armario y va metiendo las cosas que cree que va a necesitar. Los dos tienen en común que hacen la maleta pensando en el destino del viaje. Al igual que estos viajeros, los maestros también presuponemos cómo debe ser un profesor de matemáticas a partir de las buenas o malas experiencias que hayamos tenido en nuestra etapa como estudiantes.

En ocasiones, esta carga hace que la maleta sea demasiado pesada y, por ello, Comillas Airlines ofrece la posibilidad de perderla como una oportunidad inesperada de ver y aprender nuevas realidades. A nosotros nos pareció que perder la ma- 
leta era una buena excusa para renovar nuestro armario y reflexionar sobre qué tipo de profesores de matemáticas queremos ser. Sin embargo, como en todo vuelo, hay gente que reclama su equipaje siendo más reacios a llenar sus maletas de nuevos recursos y perspectivas. Haber experimentado en primera persona la pérdida de nuestro propio equipaje, nos ha hecho darnos cuenta de la importancia de tener en cuenta las maletas de nuestros alumnos y querer llenarlas de valiosos recursos y herramientas que les permitan utilizar las matemáticas como la llave para resolver situaciones cotidianas de su día a día. ¿De qué nos vale Ilenar la maleta de contenidos sin conexión, sin comprensión y sin razonamiento? No queremos que sean maletas pesadas y poco funcionales: jnos quedamos con un buen equipaje de mano que les ofrezca un amplio abanico de estrategias!

A la hora de hacer esas maletas debemos proponer escenarios de aprendizaje basados en la experiencia previa de los estudiantes o en una vivencia compartida creada en el aula. De esta manera, el alumno se incorpora a las actividades poniendo a prueba sus conocimientos previos y generando hipótesis que terminará confirmando o desmintiendo. Compartimos con M. a Antonia Canals la idea de la necesidad de introducir un interrogante para que el alumnado, a partir de su interés y su curiosidad, construya sus propios aprendizajes. Son ellos los que tienen que realizar el descubrimiento de aquello que no sabían y conocer conceptos y procedimientos nuevos. Sin embargo, no debemos confundir esto con ceder nuestro equipaje personal a nuestros alumnos: se trata de ayudarlos a que ellos mismos Ilenen de aprendizajes sus propias maletas. Por tanto, y como Fernández Bravo diría -el creador del material Numerator-, debemos "apoyar la enseñanza de la matemática en lo que el alumno desconoce, no en lo que el profesor sabe" (2007, p. 10).

Si algo no puede faltar en nuestras maletas es una idea en la que muchos autores coinciden: deben ir Ilenas de materiales que los estudiantes puedan manipular.

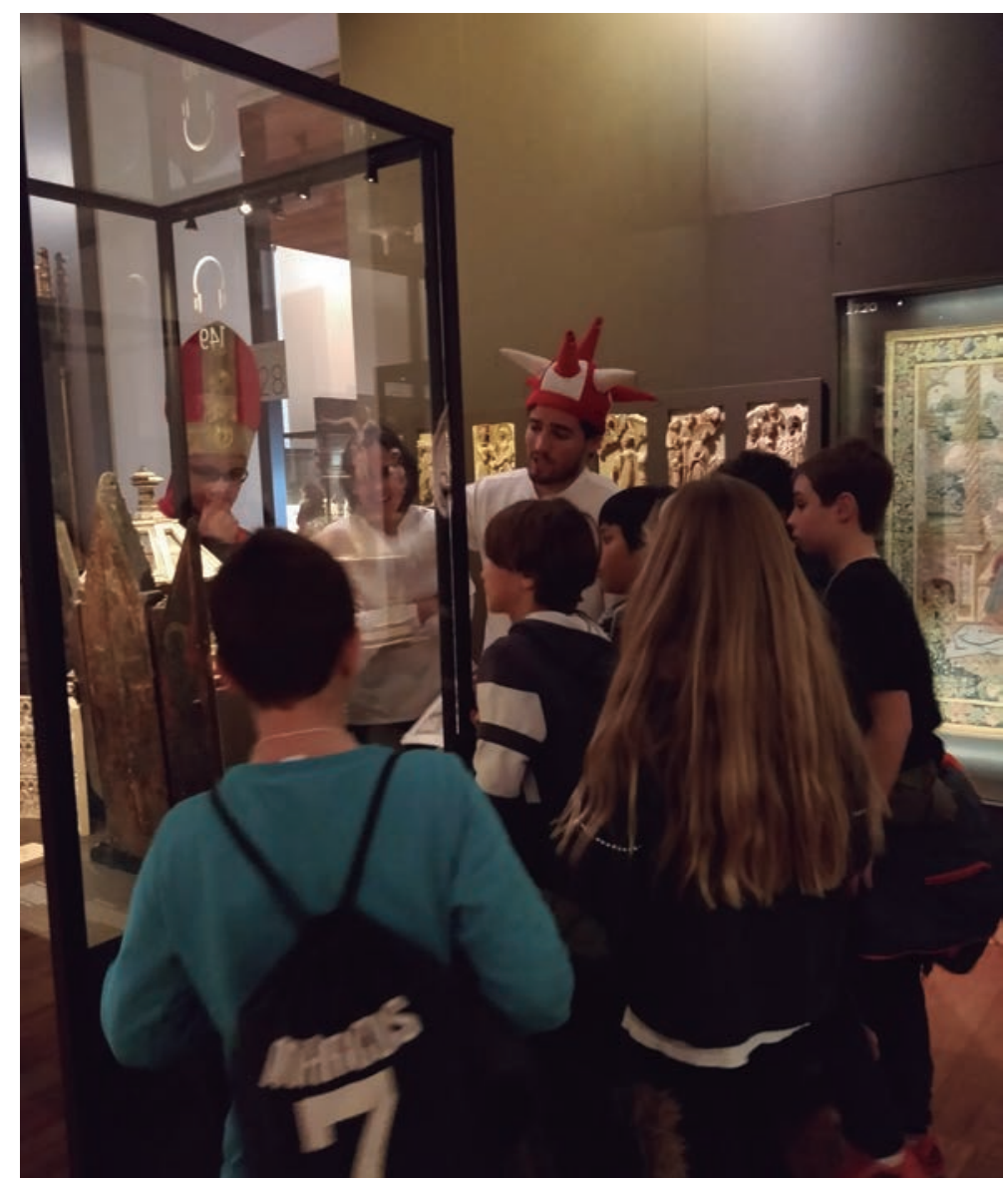

\section{Debemos proponer escenarios de aprendizaje \\ basados en la experiencia previa de los estudiantes \\ o en una vivencia comparlida en el aula. Así, el \\ alumno se incorpora a las aclividades poniendo \\ a prueba sus conocimientos previos y generando \\ hipólesis que lerminará confirmando o desmintiendo}

Hemos de ser conscientes de la dificultad de nuestros alumnos para operar con conceptos abstractos y basar nuestra enseñanza en la manipulación de materiales concretos.

\section{Vuelo internacional, matemática interdisciplinar}

Casi sin darnos cuenta, nuestro perfil de turista se ha ido convirtiendo poco a poco en uno que se asemeja al de apasionados viajeros que disfrutan más del viaje que del destino. $Y$ es que no es lo mismo ser turista que viajero. Hemos aprendido que con cada escala nuestra maleta se ha ido llenando de experiencias, aciertos 


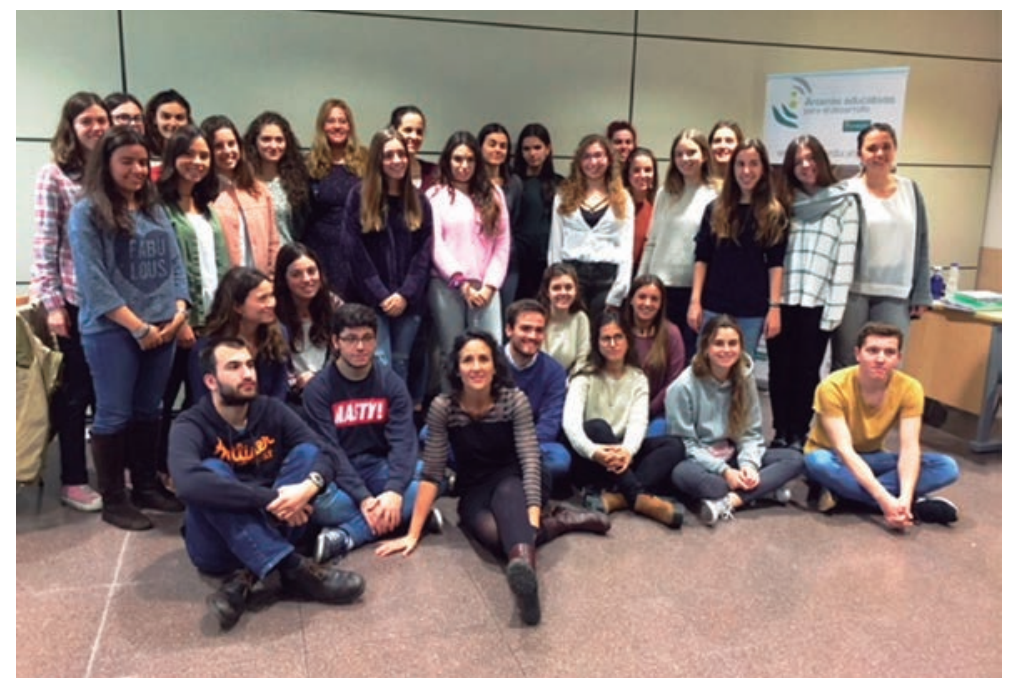

Nuestro aprendizaje como maestros de malemálicas en primaria nos ha hecho replantearnos el arigen y pensar en qué momento se perdió el sentido de aprender para converlirse en memorizar, repelir y hacer exámenes
El proyecto de la Feria de Abril con Lengua fue un reto mayor, ya que a priori no conseguíamos ver ninguna conexión entre los contenidos de ambas asignaturas. En este caso han sido los textos literarios los encargados de darnos la posibilidad de trabajar de manera paralela y conectada estas dos áreas troncales del currículo.

Por último, la colaboración de Ciencias Experimentales y Matemáticas entró en nuestra clase para enseñarnos cómo aplicar el método científico para trabajar con nuestros alumnos como auténticos investigadores. A través de Fibonacci descubrimos las matemáticas presentes en la naturaleza y pudimos profundizar en sus "números favoritos".

Tras haber visitado todos estos destinos nos hemos puesto las gafas matemáticas que nos permiten vislumbrar desde nuestra ventanilla miles de oportunidades de aprendizaje matemático.

\section{Diario de viajes, matemáticas salvajes}

En cualquier vuelo que se precie hay revistas a bordo, más concretamente en el asiento que cada pasajero tiene delante. $Y$ aunque puedes pensar que al tratarse de un vuelo matemático cambiarán las revistas por libros de texto, cuadernos de fórmulas y operaciones para practicar, lo cierto es que no. En nuestro vuelo se ha mantenido el mismo catálogo de viajes y regalos que te puedes encontrar en cualquier aerolínea. Hemos descubierto que las matemáticas están presentes tanto en una fotografía de las pirámides de Egipto, como en el $30 \%$ de descuento que te hacen si compras una colonia de 15,30€.

La única diferencia entre nuestra revista de a bordo y las demás es que la nuestra tiene las últimas páginas en blanco y solo hay escrita la frase "avistamientos matemáticos". En ellas, hemos recopilado las matemáticas más cotidianas, reformulándolas de tal manera que se han convertido en propuestas didácticas para el aula. Actividades como el Matebook y el Concurso de Fotografía Matemática, nos han permitido desarrollar nuestras habilidades para encontrar las matemáticas en cual- 
quier lugar. Doble experiencia de aprendizaje porque las hemos vivido a la vez como maestros y también como alumnos.

No solo los pasajeros llevan registro de las matemáticas que van apareciendo a lo largo del viaje, sino que la tripulación también recoge en su cuaderno de bitácora todas las huellas. No es un cuaderno a la vieja usanza sino que las TIC se cuelan en nuestra aula en forma de blog de clase o como hashtag \#matematicazas en nuestros comentarios en la red social Twitter. Nosotros, maestros, hemos aprendido una nueva forma de dejar evidencia sobre nuestro trabajo de una manera distinta a las tradicionales fichas.

\section{Matemáticas protagonistas de nuestras prácticas}

Al hacer balance sobre nuestro vuelo hemos concluido que no solo el viaje ha merecido la pena por la experiencia en sí misma, sino que ha permitido enriquecer nuestras prácticas en los centros educativos. Son innumerables las veces que los bingos matemáticos, las cartas encadenadas o las propuestas de lo que habíamos comprado en la tienda Mate-free (algunos dirán que se dice duty free) han sido protagonistas de las sesiones que preparábamos para llevar a nuestras aulas. Hemos podido vivir en primera persona cómo algunos alumnos se sorprendían al vivir "una mates divertidas" o, como ellos dicen, "aprender jugando".

Nuestro aprendizaje como maestros de matemáticas en primaria nos ha hecho replantearnos el origen y pensar en qué

\section{HEMOS HABLADO DE}

\section{Matemáticas; aprendizaje manipulativo; formación inicial; interdisciplinariedad.}

Este artículo fue solicitado por PADRES Y MAESTROS en mayo de 2018, revisado y aceptado en octubre de 2018.

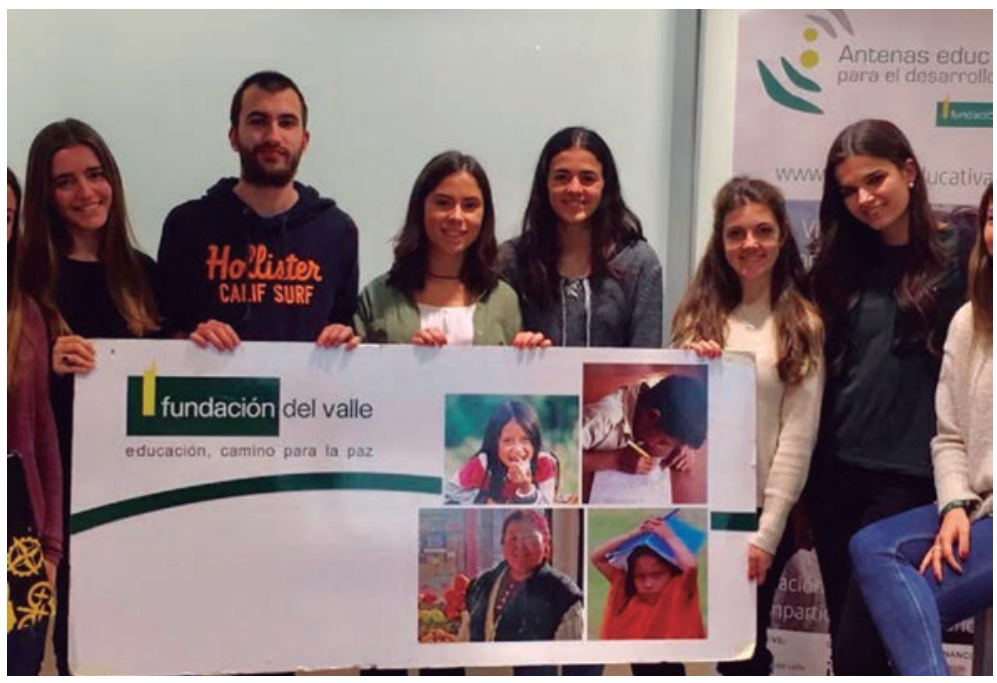

momento se perdió el sentido de aprender para convertirse en memorizar, repetir y hacer exámenes. Comillas Airlines nos ha permitido viajar a lugares que jamás habíamos pensado descubrir como el mundo de la lógica, el país del aprendizaje cooperativo, la selva de los proyectos interdisciplinares o el reino del juego. Todo ello nos ha hecho ser alumnos de una sorprendente nueva realidad que cada día se abría ante nuestros ojos para, de este modo, convertirnos en guías exploradores de muchos grupos de turistas que, aquí, en nuestros colegios, suelen ser llamados "alumnas y alumnos".

Sabemos que nuestro vuelo solo acaba de comenzar y aunque en unos años dejemos de volar con Comillas Airlines, un buen viajero nunca deja de aprender. Seguiremos en el aire por y con nuestros alumnos.

Seguiremos atentos... •

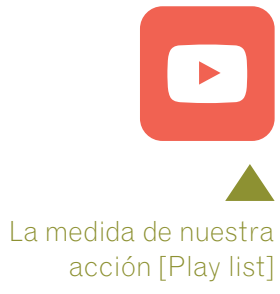

La grabación de los videos del proyecto ApS "La medida de nuestra acción" para Guatemala, fue una oportunidad para poner a prueba nuestras habilidades comunicativas acción [Play list]

\footnotetext{
(1) DARr SABER MÁS

Numerator es un juego que propone utilizar cartones y botones para modelizar nuestro sistema de numeración que es decimal y posicional. Es imprescindible que los cartones se encuentren en la posición correcta para poder operar con él, de ahí la metáfora utilizada en la preparación para el despegue de nuestro vuelo. ¿Quieres saber más?

FERnÁNDEZ Bravo, J. A. (2017). Numerator: Un juego para aprender la numeración y las cuatro operaciones. Madrid: Editorial CCS.

Universidad Pontificia Comillas. (2018). La medida de nuestra acción [Videofile - Play list]. Recuperado de https://www.youtube.com/pl aylist?list=PLwfseCnG07Tow9HkNX1mXv0mccGiQFZQ2
} 